\title{
An Integrated Interface for Proactive, Experience-Based Design Support ${ }^{*}$
}

\author{
David B. Leake \\ Computer Science \\ Department \\ Indiana University \\ Lindley Hall 215 \\ 150 S. Woodlawn Avenue \\ Bloomington, IN 47405, U.S.A. \\ leake@cs.indiana.edu
}

\author{
Larry Birnbaum \\ Intelligent Information \\ Laboratory \\ Northwestern University \\ 1890 Maple Avenue \\ Evanston, IL 60201, U.S.A. \\ birnbaum@ils.nwu.edu
}

\author{
Kristian Hammond \\ Intelligent Information \\ Laboratory \\ Northwestern University \\ 1890 Maple Avenue \\ Evanston, IL 60201, U.S.A. \\ hammond@ils.nwu.edu
}

\begin{abstract}
Many case-based reasoning systems have been developed to aid designers by providing them with libraries of prior design experiences. Traditionally, these systems are implemented as stand-alone "external memories" for the designer to query manually. This paper presents a contrasting approach that integrates proactive case retrieval into the designer's normal task processes, automatically tailoring information selection and presentation emphasis to fit changing designer needs and attention flow. The paper presents a set of principles for this integrated intelligent design support and describes their application in the Stamping Advisor, a system to support design feasibility analysis for sheet metal automotive parts. The Stamping Advisor interface proactively provides designers with relevant information to support feasibility analysis, automatically prepares their information products, and unobtrusively gathers the information needed to generate new cases to improve the quality of future support.
\end{abstract}

\section{Keywords}

Case-based reasoning, context, design, intelligent information systems, just-in-time retrieval, knowledge management

\section{INTRODUCTION}

Case-based design support systems aid designers by providing them with a "shared external memory" [13, p. 19] of prior design examples, to suggest useful solutions and warn of potential problems (for a collection of recent examples, see [14]). Such systems have proven very useful, but leave considerable burden on the designer. To use them, the designer must first take the initiative, deciding whether to in-

\footnotetext{
*Additional authors are listed at the end of this paper.
}

Permission to make digital or hard copies of all or part of this work for personal or classroom use is granted without fee provided that copies are not made or distributed for profit or commercial advantage and that copies bear this notice and the full citation on the first page. To copy otherwise, to republish, to post on servers or to redistribute to lists, requires prior specific permission and/or a fee.

IUI'01, January 14-17, 2001, Santa Fe, New Mexico, USA.

Copyright 2001 ACM 1-58113-325-1/01/0001 ..\$5.00 terrupt other tasks to query the system-often without any knowledge of the likelihood that relevant information will be available. The designer must then specify a set of retrieval features for the system to use, and, if cases are retrieved for those features, must decided whether they apply and how to apply them.

This paper describes research on reducing the user's burden by integrating case-based design support into the task process, in order to automatically provide the right information at the right times. In our approach, the system proactively presents relevant cases to warn about potential problems and propose solutions. To help the designer decide whether retrieved cases apply and how to apply them, it automatically supports the designer's relevance judgment by a three-point comparison method which we call case bracketing. In addition, it further aids case application by using task and case information to automatically generate context-based queries to search supplementary information resources. Finally, it monitors the designer's interactions to automatically prepare the designer's written work product as a byproduct of the designer's task performance.

The paper begins by presenting a set of principles for intelligent design interfaces integrating case-based support, and then illustrates how those principles have been realized in the Stamping Advisor, a tool to support feasibility analysis for sheet metal automotive parts. By integrating the task interface and CBR system, integrating cases with other information resources, and monitoring the designers' interactions to capture task-relevant information, this approach automatically provides designers with the information they need and builds up useful information for transmission and future use.

\section{PROJECT AND SYSTEM OVERVIEW}

The Stamping Advisor project aims to provide a unified interface for accessing, collecting, and applying design case information. It contains components to anticipate information needs during the design process and retrieve prior cases, a graphical interface to present information that highlights potential problems, and additional query generation and retrieval components to retrieve task-relevant information from on-line resources. 
The Stamping Advisor supports the analysis of a design by first retrieving cases for similar previous parts that suggest potential issues, providing the part descriptions, issue descriptions, and descriptions of their resolutions to the feasibility engineer, and automatically retrieving bracketing cases to help determine whether the issues apply. It uses the information about prior issues to automatically generate queries to retrieve web-based information relevant to those issues, searching on-line Ford manuals. Its final product is the report that the designer must provide at the end of feasibility analysis. Thus the system supports a designer by (1) proactively providing relevant prior cases, (2) integrating the presentation of those cases naturally into the designer's attention flow, (3) supporting the designer's case evaluation and case application, and (4) unobtrusively capturing information to generate the designer's information product.

\section{PRINCIPLES FOR INTEGRATED INTELLIGENT DESIGN SUPPORT}

The Stamping Advisor's design was guided by five principles for intelligent design support interfaces: ${ }^{1}$

- Seamless interaction. Interaction with the combined system must fit seamlessly into the designer's normal problem-solving process.

- Just-in-time, context-based retrieval. The system must proactively anticipate information needs for the current design subtask, and automatically provide the right information when it is needed, rather than placing the burden on the user to formulate requests.

- Knowledge source integration. Retrieval must bring multiple knowledge sources to bear in a coherent way, both to aid the task directly and to help determine the applicability of provided information in the current context.

- Automatic generation of information products and experience capture. The interface must generate the designer's information products, using its monitoring of task context to gather needed information, and must exploit the knowledge it gathers to automatically store new cases to improve future support.

- Cross-task integration. The interface must support integration of information across different subtasks of the design process. This integration should automatically access information about the previous tasks to provide a context for its information presentation and should produce products that can be used by downstream reasoning processes.

This paper focuses on how the design of the Stamping Advisor's user interaction contributes to the realization of these principles. The system's contributions to case-based reasoning are discussed in [9], which illustrates the system with an earlier version of the interface.

\section{THE STAMPING ADVISOR'S TASK DOMAIN}

Work on the Stamping Advisor arose from needs at the Ford Motor Company to develop an intelligent interface to assist manufacturing engineers as they consult prior parts during stamping feasibility assessment. In the design process for automotive parts, manufacturing engineers interact with design engineers to ensure manufacturing feasibility. Given a preliminary part design, manufacturing engineers produce a feasibility study identifying manufacturability issues that may affect part quality, production efficiency, or manufacturing costs. Feasibility judgments are made mainly on the basis of part geometry and material properties. For example, some shapes are difficult to make because of the stretchability of metal, which may result in unpredictable "springback" of the metal towards its original shape after stamping. The feasibility engineer's task is to identify potential problems, to justify why they are likely to occur, to estimate the resulting costs if they are not addressed, and to propose design revisions to remedy them.

A central issue in this process is how to provide feasibility engineers with the information they need. Expert feasibility engineers report that they often base their judgments on specific experiences with prior designs, but novices must begin without this resource, and even experienced engineers may lack experience with the most relevant designs for a particular part. At the start of the project, the Ford Motor Company had already captured paper records of issues and decisions and had gathered initial "seed cases" in a database, and wanted to develop effective access methods. At that point, on-line manuals and other information resources existed to aid the feasibility analysis task, but it was often time-consuming for engineers to locate the information they needed. Likewise, when engineers completed their analysis, communicating their decisions and justifications was cumbersome: The standard method for communicating their decisions was to prepare and send a paper document.

This led to our research on methods for providing feasibility engineers with two types of support. The first type is support in the form of needed information: the information they need to identify potential issues, and the supplementary information they need to evaluate those issues and support their evaluations. The second is support in the form of records of their task activity, to transmit to upstream designers - to suggest and justify design revisions-and to downstream designers - to justify the final design.

We will discuss the resulting approaches in four sections. The first three sections describe ways the system provides needed information: The issue summary interface, which integrates proactive case retrieval into the engineer's normal task process; the issue examination and case bracketing interface, which helps engineers determine the applicability of issues and repairs; and the automatic context-based query formation mechanism, which combines task characteristics with extracted case features to form queries for needed supplementary information. The fourth section describes the information collection and transmission process. We note, however, that the Stamping Advisor performs its information collection during ongoing processing as the designer uses the other interfaces.

\footnotetext{
${ }^{1}$ These principles are revised from [9].
} 


\section{THE ISSUE SUMMARY INTERFACE}

One of the goals of the Stamping Advisor project is to make the presentation of cases fit the engineer's own reasoning. This is done both through the design of the issue summary interface, and by using knowledge of the engineer's task to anticipate the engineer's information needs and proactively supply the engineer with useful information.

In the traditional feasibility analysis process, feasibility engineers evaluate parts by examining the CAD image of the part to evaluate. Interviews with feasibility engineers established that one of their reasoning styles is to scan the image sequentially, tracing around the border of the part looking for portions of the design that raise feasibility issues. The Stamping Advisor's primary interface parallels and supports this process by augmenting the CAD image of a part with automatically-generated annotations for part regions, each annotation summarizing the results of an automatic retrieval of cases for parts with similarities in those regions (e.g., the headlamp area would be annotated with a summary of retrieval results for cases involving issues for similarly-shaped headlamp regions). As shown in figure 1, the graphical interface organizes case information geometrically according to the regions of the part. To gather information relevant to each region, the Stamping Advisor performs a separate retrieval for each region, based on a coarse-grained geometric representation. Many stamping issues are associated with single regions, but issues can also involve problems related to interacting features from adjacent regions; see [9] for further discussion of the system's retrieval methods.

As the engineer scans each part region, he or she can consult the issue summaries to suggest regions requiring special scrutiny and to provide access to relevant cases. The issue summaries include the number and severity of issues found for each region, highlighted with color-coded warnings to identify the most problematic regions (a green bullet when prior cases support feasibility, yellow for limited problems, and red for more serious problems). Figure 1 shows the issue summary interface for an automobile fender. In the screen image, the bottom center box, describing the wheel opening, is marked with a yellow bullet because previous cases identified two potential issues, but both were resolved. No other problems were found, so all other bullets are green.

Based on the issue summary information, the feasibility engineer may select a region of interest to examine using the issue examination and case bracketing interface. This provides the capability to view and edit the issues suggested by prior cases. The sample window shown in Figure 2 presents an example in which two issues have been found, both associated with the trim condition of the "forming bottom." These are summarized in a menu at the top of the page, which highlights the issues with color-coded bullets (red, yellow, or green) to indicate their estimated severity. In this example, two distinct issues were found involving the trim condition, one that a prior feasibility engineer chose to disregard (indicated in green) and one more serious (indicated in yellow).

The engineer may choose not to examine an issue, in which case the prior engineer's response is added to the final report. When the engineer chooses to examine the problem, he or she may select issues from the issue menu to see more detailed information: (1) the specific aspects of the region involving issues, (2) a standardized, structured description of the problem, and (3) how it was resolved by a previous designer. For each problematic feature, the case representation contains a representation of the problem in terms of the part feature with potential problems (e.g., the "forming bottom"), the attribute of the feature causing the problem (e.g., "trim tolerance"), and a qualifier of the problem (e.g., that trim tolerance is "tight"). The case also includes a solution description. The solution may simply be to ignore the problem, or a structured description including the feature, the action (e.g., "increase" for trim tolerance), the specific action parameters (e.g., from $+1.0 /-1.0$ to $+5.0 /-1.0$ ), and optional textual rationale for the change.

When presented with this information about a relevant prior issue, the designer must decide whether the issue applies, its level of severity, whether to request a design change, and if so, what change to request. This requires assessing the applicability of the prior case and its solution. The Stamping Advisor supports this process in two ways. First, it supports a three-way comparison process between the most similar prior design with the problem, the current design, and the most similar design without the problem. Second, it automatically generates queries to search supplementary knowledge sources for information to guide the case application process. These are discussed in the two following sections.

\section{CASE BRACKETING: COMPARING ISSUES WITH BORDERING CASES}

After presenting retrieved cases, case-based decision-aids often leave to the user the task of judging their applicability. As expressed by Kolodner (p. 57, 1991), these systems serve only as memories: "we leave the real decision making to people." Yet in practice, it may be difficult for users to decide whether a given case applies, to identify the significant features, or to know the scope of changes needed to overcome its problems. For example, when assessing the severity of a problem it is useful to know whether a minor variant is likely to be successful, as well as to have guidance on which features to change. The Stamping Advisor provides this information by using its cases in a novel way. Rather than simply presenting the most similar case(s), as is customary in CBR systems, it automatically presents the user with two side-by-side bracketing cases to compare to the situation at hand: the most similar with the problem and most similar without. These cases help delineate the limits of the problem being considered. They enable to designer to determine which features are important to the issue, by noting differences between the success and failure cases, and to assess the severity of the problem, by comparing current features to the most relevant good and bad prior examples. They also suggest additional possible repairs that are implicitly contained in the case base, by presenting a similar design that would overcome the problem. Figure 2 shows the presentation of two bracketing cases for a problem in the condition of the part trim.

\section{AUTOMATIC CONTEXT-BASED QUERY FORMATION}

To further support the designer's evaluation of problem severity and selection of responses, the issue examination interface also allows search of on-line resources (e.g., the electronic design manuals on Ford's internal network). Using knowledge of the user's task and task context, determined 


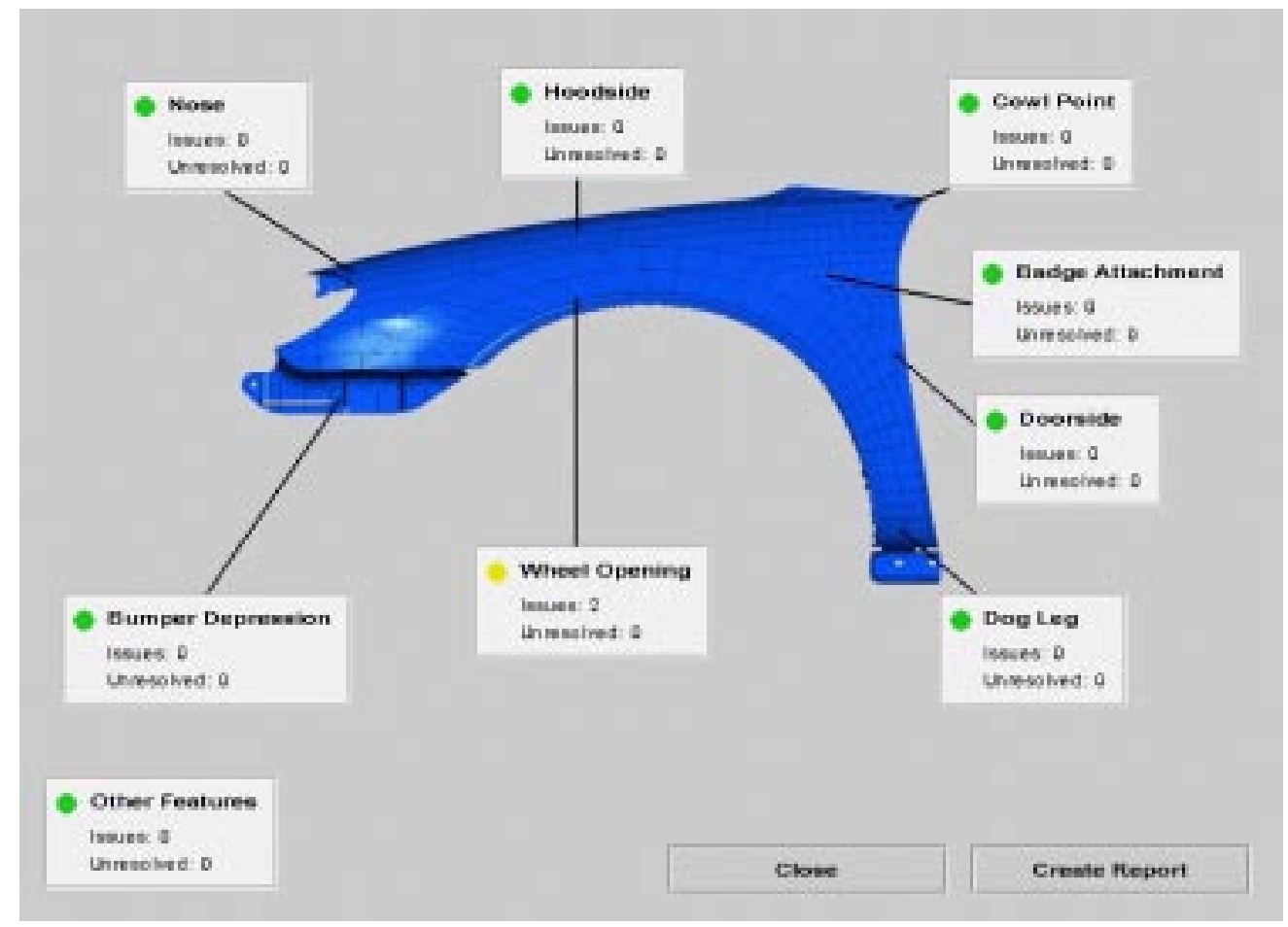

Figure 1: Screen image from the Stamping Advisor's issue summary screen.

by an overall task model and the cases under consideration, the system automatically formulates targeted queries to go against any documents indexed by standard search engines.

The Stamping Advisor builds queries using two types of information. The first ("part search terms"), is a set of basic descriptive features relevant to the domain, including the car model, part region, and name of the problematic feature. The second ("problem search terms"), is a set of problem attributes extracted from the problem description of the retrieved case. The system forms its queries by extracting textual strings for each piece of information from the current task context (the record of the part being designed and the prior case suggesting a problem): the name of the vehicle for which the current part is being designed (e.g., "Ranger"), the name of the current part region (e.g., "wheel opening"), the problematic feature (e.g., "forming bottom"), and the problem in question (e.g., poor trim condition). Upon the feasibility engineer's request, this query is used to search for relevant guidelines in on-line manuals. Before initiating a search, the engineer can request that the query be focused only on similar parts or similar problems, and can edit the query text as desired (e.g., replacing "Ranger" to compare the styling on a different line of car). Sample query options are shown at the bottom of Figure 2.

Once created, the query can be passed to any Internet search engine to search selected resources. The current implementation uses the system Verity to index on-line Ford documents such the Ford Style Guide. The search engine result - a ranked list of matches - is presented to the feasibility engineer to select documents to retrieve. Evaluating and refining this query-generation method are topics for future research, but informal tests suggest that it is sufficient to retrieve relevant information in this domain, which has a fairly standardized lexicon and for which the indexed documents are all task-focused resources.

\section{INFORMATION COLLECTION AND TRANSMISSION}

Most case-based design support tools have a natural goal: aiding a designer in his or her direct task. However, in industrial settings, the designer's task is only one step in an extended process. In stamping design, the design is refined though an iterative cycle of critiques by the feasibility engineer, which must be conveyed to upstream designers so that they can revise the design or reject changes. When a design is finalized, the designer must pass information to downstream design team members who evaluate and apply the final design. These communications require generation of a record to contain information on issues and the feasibility engineer's justifications for why they matter (or do not matter), and how they were resolved. Thus the effectiveness of the system depends not only on its interaction with the current engineer, but also on its interaction with up- and downstream designers - as well as its support of temporallydelayed communication with future designers who may wish to consult the current designer's reasoning in the future. As a result, building up a record of the designer's decisionmaking, generating the final work product for communication downstream, and capturing new cases are all central system tasks. As the Stamping Advisor is used, the system automatically records information to build up task records as a byproduct of the basic feasibility assessment task. 


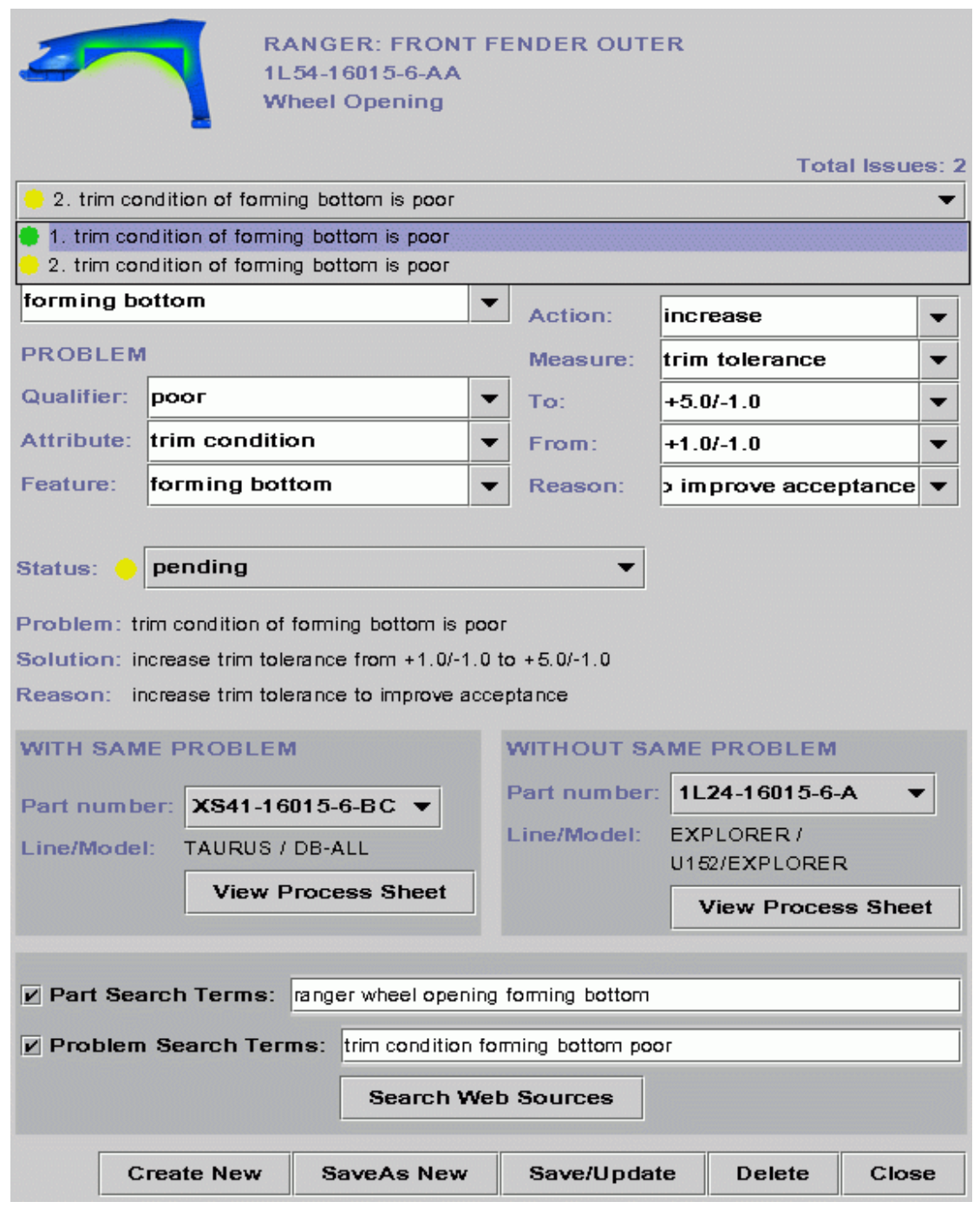

Figure 2: The issue examination and bracketing interface, presenting wheel opening issues. The current part is bracketed by the closest problem- and non-problem cases for the current issue.

\subsection{Capturing issue descriptions and responses}

As the system helps feasibility engineers to identify issues and determine appropriate responses, it also aids them in describing their conclusions. To facilitate information entry, it automatically fills in a record with information extracted from the current subtask and context. This includes information from the current part record (e.g., part number), as well as predicted problems (from prior cases), and their previous solutions. The designer may simply accept these descriptions, or may enter or adjust additional information using pulldown menus for different fields. Each menu provides options from a standard vocabulary of problem and feature descriptions. This facilitates problem entry and also assures that problems will be described in a standardized vocabulary, facilitating future retrievals by the case retrieval mechanism.

To increase flexibility, the information from the menus may also be supplemented with free-text information if needed. This supplementary information could potentially be mined to identify new vocabulary elements as the system is used, but this has not been explored in the current system

\subsection{The report and case generators}

At the close of the feasibility assessment process, the system uses information gathered throughout the task process to generate a Final Report Document to be sent up- or downstream, replacing documentation generated by hand. In the task model developed for the Stamping Advisor, the information needed by the evaluation process is: (1) the part being examined, (2) the issues considered, (3) how they were disposed of, and (4) the substantiating evidence relevant to the issues and decisions (e.g., the cases with the most similar regions with problems and how the previous designers disposed of those issues, and the cases with the most similar regions without issues). The Final Report Document (FRD) is produced as the by-product of the user's decisionmaking and requires no additional effort on his or her part beyond recording the previously-described decisions (which are a small subset of the information that previously needed 
to be entered on paper reports to be sent downstream). A sample FRD is shown in Figure 3. The FRD highlights each region under consideration in yellow on a small part image, and presents part features, issues, and bracketing case information. The document is an html page containing links to the case records for the bracketing cases considered by the feasibility engineer, so that downstream designers can examine those cases to clarify questions.

Although the Stamping Advisor project focuses only on feasibility analysis, it illustrates a general approach for gathering data at each phase of a design process, to make the growing record available to other processes in order to support related reasoning and for them to add to this record for future use. As an example of how this could be used, consider the development of part and process designs for stamped automobile body parts, which involves four design phases: engineering design (of the initial shape), feasibility analysis, die process design (to determine how stamping will be done, and with how many dies), and die design. Cases storing information captured or augmented during each design phase can provide a vehicle for the automatic accumulation of information and communication up- and downstream, for designs, issues, revisions, and rationale. Thus automatic case capture also provides a medium for capturing information for automatic transmission and use at multiple points in the design process.

This type of information is particularly important for $d e$ signing for manufacturability, which aims to increase design and manufacturing efficiency by taking manufacturing issues into account from the beginning of the design process. Design for manufacturability requires bringing lessons to bear across different design phases, and is often impeded by lack of communication between designers involved in different phases. Consequently, design aids that automatically share information across design phases promise important benefits. For example, problems revealed during the die design process for a part may suggest new lessons about which shapes are hard to manufacture and why. The resulting cases - generated during the die design phase - can then be applied during future feasibility analysis, in order to warn the engineering designer so that problematic shapes can be avoided.

\section{PERSPECTIVE}

\subsection{User Response}

The Stamping Advisor was developed in consultation with Ford feasibility engineers, to understand their task process and assure that the system design responded to their information needs. The final interface design, presented here, was tested by senior engineers from Ford's stamping operations divisions in Dearborn, Michigan and from multiple locations in Germany, who tested system functionality on a set of sample parts. Although their experiences with numerous systems and system changes make them skeptical of new software tools, their responses were uniformly positive. After trying the system, one gave it the high praise of describing it as "a system we'd actually use."

\subsection{Relationship to Previous Research}

As described previously, the design of the Stamping Advisor was shaped by five principles for developing intelligent decision support systems for design. Its methods con- tribute to a number of research and applications areas, including case-based design support, design critiquing, knowledge management, and proactive retrieval.

\subsubsection{Case-based design support}

Case-based design support has been the subject of extensive study. For reasons of space, this section will focus on the Stamping Advisor's contributions to user interface issues for case-based support systems, referring the reader to [9] for a discussion centered on case-based reasoning issues.

Case-based design-aiding systems are often conceived as retrieval systems - "external memories"-for the user to invoke when needed. To retrieve cases, the user inputs a set of indices to guide case retrieval. For example, the SAM system [12] for architectural design allows users to retrieve cases by entering a building's name, filling out a form with important features, or entering a text string to match with the text in stored cases. In the FABEL system [5], the user first selects a retrieval method and then provides the data that method requires. This differs dramatically from the Stamping Advisor's approach, which automatically selects features in order to perform proactive retrieval.

Another contribution concerns the flexible focus of the Stamping Advisor's retrieval process. Previous systems that retrieve design images, such as $[5,6]$, do not attempt to reflect specific goals for using the retrievals. Consequently, they must retrieve based on overall similarity, rather than selectively focusing on task-relevant features. For example, when designing a fender, standard image-based approaches would always try to retrieve the case for the most similar prior fender. However, if the engineer is assessing the headlamp opening, the right retrievals will be fenders with similar headlamp openings, even if their overall styles are quite different (e.g., if a similar headlamp design was previously used in a truck rather than a car).

In case-based design aids, support for case application usually focuses on helping the user to interactively revise a design (e.g., [11]); evaluating the applicability of a proposed prior design - and gathering the information needed enable that evaluation - are left to the user. For feasibility analysis, however, this evaluation is crucial and may be quite subtle, requiring support. The three-point comparison supported by the Stamping Advisor's bracketing interface is a novel approach to supporting interactive case evaluation, and is applicable to a wide range of task domains.

The Stamping Advisor also differs from other case-based design aids by using knowledge about the task and information from retrieved cases to automatically generate search engine queries, to further assist the designer in understanding and applying the case at hand. (Cf. [16], which proposes using the complete textual descriptions of cases as input to an IR system in order to find additional similar cases.) The spirit of this approach is similar to the automatic contextbased query formation of Watson [3]. However, that system must generate a context description by hypothesizing the important points in an open document, while the Stamping Advisor has access to much more precise contextual information because of its tight task integration.

The Stamping Advisor's task integration also helps to address the classic CBR problem of how to build up an initial case library. Although the Stamping Advisor system is provided with an initial "seed case base," even if no cases were available in the system case library, the system would 


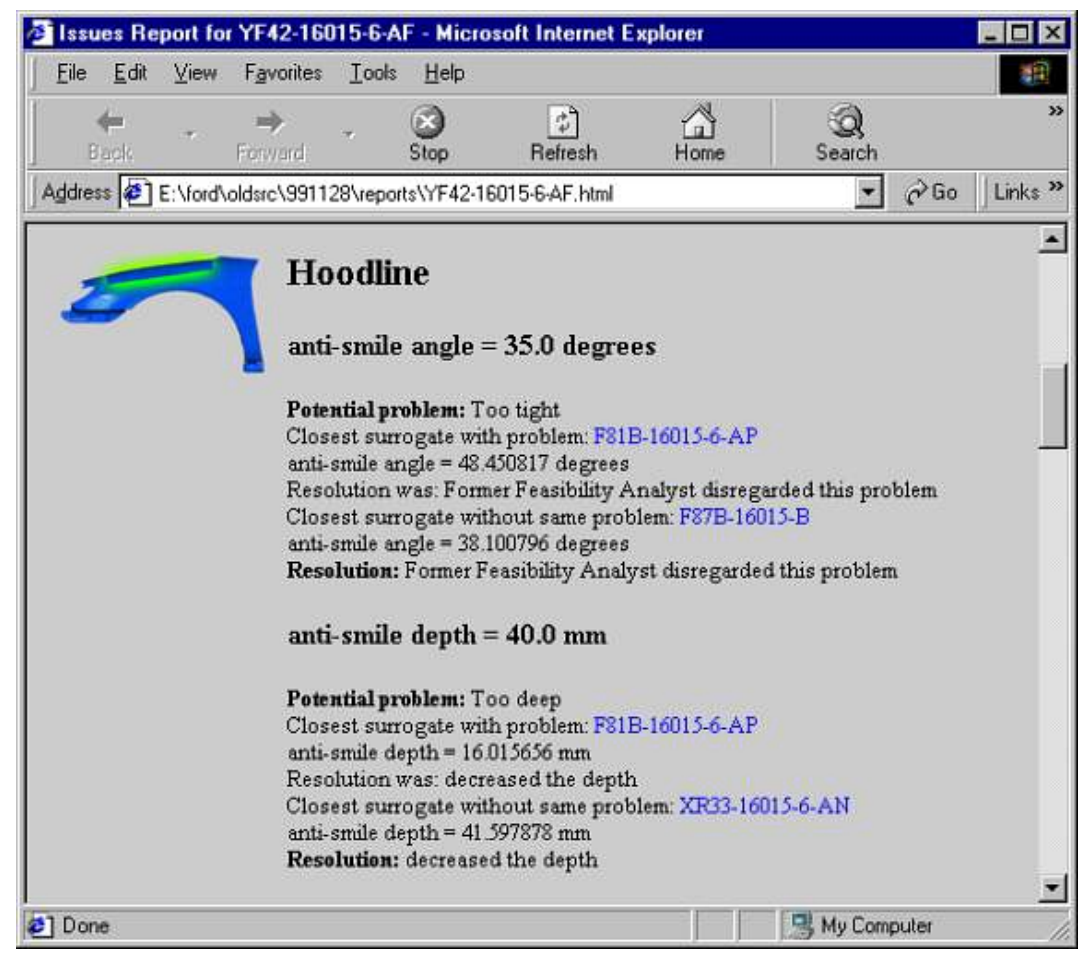

Figure 3: A final report document.

be useful as a convenient interface for generating the work product of the feasibility assessment process (the Final Report Document) and aiding in searching on-line resources. This provides the incentive to use the Stamping Advisor while its case library is being built up, providing a stream of new cases for the system to capture during use.

\subsubsection{Design critiquing}

The Stamping Advisor's task of identifying potential design issues relates to research on design environments with embedded critics. As discussed in [4], research on critiquing systems has shown a delicate balance between passive (userinvoked) and active critics: user-invoked aids are often invoked too late, but active advice may be obtrusive and disrupt the designer's work flow. The Stamping Advisor's issue summary interface makes information summaries accessible at a glance during the engineer's part-scanning process, but does not intrude on the engineer's own reasoning.

An interesting question is how methods like the Stamping Advisor's could be integrated with other design support tools, and at other points in the design process, to provide experience-based guidance wherever it is needed. For example, ideally the system would be integrated with the CAD system used by the designer during initial design steps, to immediately warn of possible problems. Likewise, the system could be integrated with other tools to help the designer respond to the problems suggested by cases, e.g., to explore design variants with model-based visualization tools [17]. However, it is important to note that the ability to present prior cases and outcomes to the user provides useful support even when no models are available.

\subsubsection{Knowledge management}

Case-based reasoning is now receiving attention as a technology for knowledge management [1]. Although current knowledge management systems are designed with particular tasks in mind, it is usually the user who must make the actual link between tasks and information: the user decides whether to seek information, the type of information to seek, and the tools with which to access that information, as well as often taking extra steps to document the task process for capture. Likewise, lessons learned systems play an important role in numerous organizations [2], but are also often conceived of as passive resources that require manual information entry and user-initiated retrievals. The approach described in this paper contributes by instead making knowledge capture and access automatic byproducts of using the interface. Leake et al. [10] provide additional discussion of the project from a knowledge management perspective.

\subsubsection{Just-in-time retrieval and support}

The Stamping Advisor belongs to the class of intelligent information systems aimed at providing proactive, "just-intime" information access for individuals engaged in tasks. Examples of this approach include context-sensitive retrieval projects such as Watson [3], the remembrance agent [15], and Microsoft's Lumiere [7]. However, the Stamping Advisor's integration into a very focussed task environment makes it possible to tailor the interface and interaction towards a precise task sequence and well-understood user needs.

We believe that the tenets and integrated methods developed in the project, when adapted to the specifics of other design problems, will prove useful in an extensive range of design tasks. 


\section{CONCLUSION}

The Stamping Advisor project illustrates a set of principles for integrating interactive case-based support systems into the larger task context, and for exploiting that integration to provide improved support. The system allows designers to perform their natural scanning process while providing just-in-time access to the right cases, and determines information sources, indices, and presentation emphasis to fit designer needs and attention flow. When the feasibility engineer's subtask is detecting issues in part regions, the system presents the parts with issues in similar regions; when the subtask is determining applicability of an issue, it presents the cases with the most similar regions with and without the issue; and when the subtask is applying the suggested revisions from prior cases, it generates queries to retrieve relevant guidelines. During this process, the system unobtrusively gathers, captures, and transmits information, to automatically generate the feasibility engineer's work product and support communication up- and down-stream. The prototype Stamping Advisor system was received enthusiastically by prospective users.

The central lessons from this project are the value of integrating case-based design support systems into the larger task and information context, and the methods developed for achieving that integration. We see opportunities to continue to strengthen the integration of the current system into the task, for example, by fully integrating the Stamping Advisor into the initial CAD design process, to immediately warn the original designer if prior cases predict problems while the original design is being generated. We believe that integrated, proactive case-based support systems are a promising basis for intelligent interfaces that not only provide guidance from experience and other sources, but also capture and transmit useful information for future use.

\section{ACKNOWLEDGMENTS}

We would like to thank Lin Qiu for his contributions to the Stamping Advisor system. This research was supported in part by the Ford Motor Company under award 0970-355A200. David Leake's research was supported in part by NASA under award No NCC 2-1035.

\section{ADDITIONAL AUTHORS}

Additional authors: Cameron Marlow, The Media Laboratory, Building E15, MIT, 77 Massachusetts Avenue, Cambridge, MA 02139, cameron@media.mit.edu, and Hao Yang PDC, MD 270, GB-D68, Vehicle Operations, Ford Motor Company, 21500 Oakwood Boulevard, Dearborn, MI 48124, hyang1@ford.com.

\section{REFERENCES}

[1] D. Aha, I. Becerra-Fernandez, F. Maurer, and H. Muñoz-Avila, editors. Proceedings of the AAAI-99 Workshop on Exploring Synergies of Knowledge Management and Case-Based Reasoning. AAAI Press, Menlo Park, 1999.

[2] D. Aha and R. Weber, editors. Proceedings of the AAAI-2000 Workshop on Intelligent Lessons Learned Systems. AAAI Press, Menlo Park, 2000.

[3] J. Budzik and K. Hammond. User interactions with everyday applications as context for just-in-time information access. In Proceedings of the 2000 International Conference on Intelligent User Interfaces, pages 44-51, 2000.

[4] G. Fischer, K. Nakakoji, J. Ostwald, G. Stahl, and T. Sumner. Embedding critics in design environments. In M. Maybury and W. Wahlster, editors, Readings in Intelligent User Interfaces, pages 537-561. Morgan Kaufmann, San Francisco, 1998.

[5] F. Gebhardt, A. Voß, W. Gräther, and B. Schmidt-Belz. Reasoning with complex cases. Kluwer, Boston, 1997.

[6] M. Gross, C. Zimring, and E. Do. Using diagrams to access a case library of architectural designs. In Artificial Intelligence in Design '94, pages 129-144. Kluwer, 1994.

[7] E. Horvitz, J. Breese, D. Heckerman, D. Hovel, and K. Rommelse. The Lumiere project: Bayesian user modeling for inferring the goals and needs of software users. In Proceedings of the Fourteenth Conference on Uncertainty in Artificial Intelligence, pages 256-265, San Francisco, July 1998. Morgan Kaufmann.

[8] J. Kolodner. Improving human decision making through case-based decision aiding. The AI Magazine, 12(2):52-68, Summer 1991.

[9] D. Leake, L. Birnbaum, K. Hammond, C. Marlow, and $\mathrm{H}$. Yang. Integrating diverse information resources in a case-based design environment. Engineering Applications of Artificial Intelligence, 12(6):705-716, 1999.

[10] D. Leake, L. Birnbaum, K. Hammond, C. Marlow, and H. Yang. Task-based knowledge management. In Proceedings of the AAAI-99 Workshop on Exploring Synergies of Knowledge Management and Case-Based Reasoning, pages 35-39. AAAI Press, 1999.

[11] C. Lottaz, R. Stalker, and I. Smith. Constraint solving and preference activation for interactive design. Artificial Intelligence for Engineering Design, Analysis and Manufacturing, 12(1):13-27, January 1998.

[12] M. Maher. SAM: A multimedia case library of structural designs. In Y.-T. Liu, J.-Y. Tsou, and J.-H. Hou, editors, Proceedings of CAADRIA '97, pages 5-14, 1997.

[13] M. Pearce, A. Goel, J. Kolodner, C. Zimring, L. Sentosa, and R. Billington. Case-based design support: A case study in architectural design. IEEE Expert, 7(5):14-20, 1992.

[14] P. Pu and M. L. Maher. Issues And Applications Of Case-based Reasoning to Design. Lawrence Erlbaum, Mahwah, NJ, 1997.

[15] B. Rhodes. Margin notes: Building a contextually aware associative memory. In Proceedings of the 2000 International Conference on Intelligent User Interfaces, pages 219-224, 2000.

[16] E. Rissland and J. Daniels. The synergistic application of CBR to IR. Artificial Intelligence Review, 10:441-475, 1996.

[17] L. Tweedie, R. Spence, H. Dawkes, and H. Su. Externalising abstract mathematical models. In Proceedings of CHI '96: Human Factors in Computing Systems, pages 406-412. ACM, April 1996. 